\title{
Erwerbspersonen- und Arbeitsvolumenprojektionen bis ins Jahr 2060
}

\author{
Daniel Ehing $\cdot$ Stefan Moog
}

Angenommen: 23. November 2012 / Online publiziert: 6. Dezember 2012

(C) Institut für Arbeitsmarkt- und Berufsforschung 2012

Zusammenfassung Diese Arbeit analysiert die Auswirkungen des demografischen Wandels auf das Arbeitsangebot und leitet auf Basis verschiedener Szenarien Politikempfehlungen ab, um dem demografisch bedingten Arbeitskräfterückgang zu begegnen. In einem ersten Schritt werden hierbei Erwerbspersonenprojektion anhand eines KohortenKomponenten-Modells vorgenommen und durch verschiedene Modellrechnungen ergänzt. Neben einer Abschätzung der Erwerbspersonenzahl bei einer Erhöhung des effektiven Renteneintrittsalters werden die Konsequenzen eines früheren Erwerbseintritts, einer steigenden Erwerbsbeteiligung der über 60-Jährigen, einer verstärkten Erwerbsbeteiligung von Menschen mit Migrationshintergrund sowie einer erhöhten Erwerbsbeteiligung der Frauen untersucht. In einem zweiten Schritt werden die Effekte einer sinkenden Erwerbslosenquote sowie einer steigenden Arbeitszeit je Erwerbstätigen betrachtet, um so Rückschlüsse über das zukünftige Arbeitsvolumen zu ziehen. Unsere Ergebnisse legen nahe, dass eine hinreichende Stabilisierung des jährlich geleisteten Arbeitsvolumens möglich ist, so dass bereits geringe Produktivitätssteigerungen dafür ausreichen werden, das Bruttoinlandsprodukt je Einwohner auf dem heutigen Niveau zu stabilisieren.

Schlüsselwörter Erwerbspersonenprojektion ·

Kohorten-Komponenten-Modell .

Arbeitsvolumenprojektion · Produktivität

JEL Klassifikationen $\mathrm{J} 11 \cdot \mathrm{J} 21$

D. Ehing $(\bowtie) \cdot$ S. Moog

Forschungszentrum Generationenverträge, 79098 Freiburg, Deutschland

e-mail: Daniel.Ehing@vwl.uni-freiburg.de
Labor force- and work volume-projections until 2060

Abstract This paper analyzes the impact of demographic change on the labor force in Germany. First, to account for observable trends in labor force participation a cohortcomponent model is applied to project the future development of the labor force. Based on this trend scenario we assess the impact of several policy options to counteract the demographic decline in labor supply. The policy options analyzed include an increase in the effective retirement age, a decline in the age of labor market entry, an increasing labor force participation of the elderly, raising the labor force participation of immigrants as well as a further increase in female participation rates. Second, the effects of a declining unemployment rate as well as the effects of an increase in the hours worked are examined, focusing on the overall annual work volume. Our results suggest that the annual work volume can be stabilized sufficiently. Therefore future productivity gains do not have to be eroded substantially in order to maintain the GDP per capita level of the base year 2010 .

\section{Einleitung}

Als Folge des demografischen Wandels sieht sich die deutsche Volkswirtschaft in den kommenden Jahren massiven Veränderungen gegenüber. Angesichts des prognostizierten Anstiegs des Altenquotienten von 34 in 2008 auf etwa 52 im Jahr 2030 (Statistisches Bundesamt 2009a, S. 39ff.) sind Erhöhungen der Beitragssätze oder Absenkungen der Leistungsniveaus zur Sicherstellung der langfristigen Finanzierbarkeit der umlagefinanzierten Sozialversicherungen unumgänglich. Neben seinen Konsequenzen für die sozialen Sicherungssysteme wird der demografische Wandel sich aller- 
dings auch direkt auf die Unternehmen auswirken und insbesondere deren Personalpolitik vor neue Herausforderungen stellen. Zählten im Jahr 2009 noch 43 Millionen Menschen zu den Erwerbspersonen (Statistisches Bundesamt 2011b), so wird diese Zahl je nach zugrunde gelegten Annahmen zur Bevölkerungsentwicklung und Erwerbsbeteiligung bis zum Jahr 2030 auf 37 bis 41 Millionen Personen zurückgehen. ${ }^{1}$ Dieser Rückgang hat zum einen zur Konsequenz, dass zukünftig bis zu einem Drittel des jährlichen Produktivitätszuwachses allein dafür benötigt wird, das Bruttoinlandsprodukt je Einwohner auf dem heutigen Niveau aufrecht zu erhalten (Börsch-Supan 2003, S. 13). Zum anderen bedeutet diese Entwicklung allerdings auch, dass sich der Wettbewerb um qualifizierte Arbeitnehmer zukünftig weiter verschärfen wird.

Anknüpfend an bereits existierende Arbeiten werden im vorliegenden Beitrag verschiedene Szenarien zur zukünftigen Entwicklung der Anzahl der Erwerbspersonen und des Arbeitszeitvolumens betrachtet, um auf dieser Basis Politikempfehlungen dahingehend abzuleiten, welche Weichenstellungen in den kommenden Jahren zu treffen sind, um dem demografisch bedingten Arbeitskräfterückgang zu begegnen. Mittels der von Burniaux et al. (2004, S. 27ff.) entwickelten und von der Europäischen Kommission (2006, S. 175ff.) verfeinerten Kohorten-Komponenten-Methode wird hierzu zunächst ein Szenario modelliert, welches neben der Berücksichtigung der Bevölkerungsentwicklung auch die beobachtbare kohorten- und geschlechtsspezifische Dynamik bei der Erwerbsbeteiligung erfasst. ${ }^{2}$ Aufbauend darauf werden dann verschiedene Szenarien modelliert: Neben einer Abschätzung der Erwerbspersonenzahl bei einer Erhöhung des effektiven Renteneintrittsalters werden die Konsequenzen eines früheren Erwerbseintritts, einer steigenden Erwerbsbeteiligung der über 60-Jährigen, einer verstärkten Erwerbsbeteiligung von Menschen mit Migrationshintergrund sowie einer erhöhten Erwerbsbeteiligung der Frauen untersucht.

\footnotetext{
${ }^{1}$ Gemäß der Primärvariante (Maximalvariante) des Statistischen Bundesamtes (2009b, S. 10) beträgt die Zahl der Erwerbspersonen im Jahr 2030 ca. 37,7 (40,7) Millionen. Je nach Bevölkerungsentwicklung liegt die Zahl der Erwerbspersonen im Jahr 2030 bei Werding (2011, S. 8) und dem SVR (2011, S. 96) zwischen 37,6 und 40,2 Millionen, wobei sich in der Basisvariante ein Wert von 38,9 Millionen Personen ergibt. Börsch-Supan und Wilke (2009, S. 37) beziffern die Zahl der Erwerbspersonen in 2030 in einem pessimistischen Szenario auf 38 Millionen und in einem optimistischen Szenario auf 40 Millionen. Bei Bomsdorf (2008, S. 143ff) liegt die Zahl der Erwerbspersonen je nach unterstellter Regelaltersgrenze in der gesetzlichen Rentenversicherung (67 bzw. 70 Jahre) und Erwerbsbeteiligung der Frauen zwischen 37 und 39,5 Millionen. Im Basisszenario von Bonin et al. (2011, S. 13) beläuft sich die Zahl der Erwerbspersonen in 2025 auf 41 Millionen, wobei sich je nach Szenario zur Entwicklung der Erwerbsbeteiligung eine Zunahme auf bis zu 42,5 Millionen Personen ergibt.

${ }^{2}$ Vorliegende Arbeit kommt hierdurch den Modellrechnungen von Werding (2011, S. 5ff.) am nächsten.
}

Neben der Zahl der Erwerbspersonen (extensives Arbeitsangebot) wird das zukünftige Arbeitsvolumen auch von der von der Arbeitszeit je Erwerbstätigen (intensives Arbeitsangebot) und der Erwerbslosenquote beeinflusst. Daher werden aufbauend auf der Erwerbspersonenprojektion im vorliegenden Beitrag auch die Auswirkungen des demografischen Wandels auf die zukünftige Entwicklung des Arbeitszeitvolumens untersucht. Als mögliche Szenarien zur Sicherung des Arbeitskräftepotentials werden dabei eine Verringerung der Erwerbslosenquote sowie eine Erhöhung der Arbeitszeit über eine Verringerung der Teilzeitbeschäftigung als auch über eine Ausweitung der Arbeitszeit für Teilzeitbeschäftigte betrachtet. Abschließend werden diese Arbeitszeitszenarien dann mit Hinblick auf die Entwicklung des Bruttoinlandsprodukts je Einwohner bei unterstellter konstanter Produktivität beleuchtet. So kann gezeigt werden, in welcher Höhe zukünftige Produktivitätszuwächse zur Absicherung des Wohlstandsniveaus von heute nötig sind.

Im Weiteren ist der vorliegende Beitrag wie folgt gegliedert: Abschn. 2.1 gibt einen Überblick über das Referenzszenario der Demografie, das dieser Arbeit zugrunde liegt. Abschnitt 2.2 stellt die Ausgangsdaten sowie das Projektionsmodell vor, dessen Ergebnisse und Modifikationen in Abschn. 3 diskutiert werden. Aufbauend darauf wird in Abschn. 4 der Einfluss einer erhöhten Jahresarbeitszeit sowie die damit implizierte Entwicklung des Bruttoinlandsprodukts je Einwohner bei konstanter Produktivität berechnet. Abschnitt 5 schließt mit einem Fazit.

\section{Datenbasis und Methodik}

Die projizierte Zahl der zukünftigen Erwerbspersonen hängt direkt mit der zugrunde liegenden Bevölkerungsvorausberechnung zusammen. Diese wird in Abschn. 2.1 erläutert. Aufbauend darauf wird in Abschn. 2.2 das KohortenKomponenten-Modell sowie die Datenbasis, die zur Projektion der zukünftigen Erwerbsbeteiligung herangezogen wird, beleuchtet.

\subsection{Demografie}

Für die Projektion der zukünftigen Bevölkerungsentwicklung wurde das von Bonin (2001, S. 245ff.) beschriebene demografische Modell zugrunde gelegt. In Anlehnung an die Annahmen der „mittleren“ Bevölkerung der 12. koordinierten Bevölkerungsvorausberechnung des Statistischen Bundesamts (2009a, S. 37) unterstellt das Standardszenario der vorliegenden Arbeit, dass die zusammengefasste Geburtenziffer auf dem heutigen Niveau von annähernd 1,4 Kindern pro Frau verharrt und die Lebenserwartung gemäß der 
Tab. 1 Bevölkerungsvorausberechnung bis ins Jahr 2060

\begin{tabular}{|c|c|c|c|c|c|c|c|}
\hline \multirow[t]{2}{*}{ Art der Nachweisung } & & \multicolumn{6}{|c|}{31.12 des Jahres } \\
\hline & & 2009 & 2020 & 2030 & 2040 & 2050 & 2060 \\
\hline \multirow[t]{2}{*}{ Bevölkerungsstand } & in 1000 & 82002 & 80367 & 78514 & 75760 & 72350 & 68540 \\
\hline & $2009=100$ & 100,0 & 98,0 & 95,7 & 92,4 & 88,2 & 83,6 \\
\hline \multirow[t]{3}{*}{ Unter 20 Jahre } & in 1000 & 15619 & 13803 & 13191 & 12225 & 11232 & 10688 \\
\hline & in \% der Bevölkerung & 19,0 & 17,2 & 16,8 & 16,1 & 15,5 & 15,6 \\
\hline & $2009=100$ & 100,0 & 88,4 & 84,5 & 78,3 & 71,9 & 68,4 \\
\hline \multirow[t]{3}{*}{20 bis unter 65 Jahre } & in 1000 & 49655 & 48208 & 43544 & 39794 & 37850 & 34990 \\
\hline & in \% der Bevölkerung & 60,6 & 60,0 & 55,5 & 52,5 & 52,3 & 51,1 \\
\hline & $2009=100$ & 100,0 & 97,1 & 87,7 & 80,1 & 76,2 & 70,5 \\
\hline \multirow[t]{3}{*}{65 Jahre und älter } & in 1000 & 16729 & 18355 & 21778 & 23742 & 23267 & 22862 \\
\hline & in \% der Bevölkerung & 20,4 & 22,8 & 27,7 & 31,3 & 32,2 & 33,4 \\
\hline & $2009=100$ & 100,0 & 109,7 & 130,2 & 141,9 & 139,1 & 136,7 \\
\hline \multicolumn{8}{|c|}{ Jugend-, Alten-, Gesamtquotient: Auf Einhundert 20- bis unter 65-Jährige kommen } \\
\hline \multicolumn{2}{|c|}{ Unter 20-Jährige } & 31,5 & 28,6 & 30,3 & 30,7 & 29,7 & 30,5 \\
\hline \multicolumn{2}{|l|}{ 65-Jährige und Ältere } & 33,7 & 38,1 & 50,0 & 59,7 & 61,5 & 65,3 \\
\hline \multicolumn{2}{|l|}{ Zusammen } & 65,1 & 66,7 & 80,3 & 90,4 & 91,1 & 95,9 \\
\hline
\end{tabular}

Quelle: Eigene Berechnungen in Anlehnung an Statistisches Bundesamt (2009a)

Periodensterbetafel von Männern (Frauen) von heute 77,2 $(82,4)$ bis 2060 auf 85,0 $(89,2)$ Jahre ansteigt. Hinsichtlich der Außenwanderung wird ein langfristiger Wanderungsgewinn von jährlich 150.000 Personen ab dem Jahr 2017 unterstellt, wobei die Sockelwanderung hierbei 600.000 Personen beträgt. $^{3}$

Auf Grundlage der getroffenen Annahmen ist bis zum Jahr 2060 mit einem Bevölkerungsrückgang von heute 82 auf 69 Millionen Personen zu rechnen (siehe Tab. 1). Wesentlich dramatischer fällt jedoch die gleichzeitige Alterung der Bevölkerung aus: Betrug der Altenquotient, d.h. die Anzahl der über 65-jährigen Personen pro 100 Personen im Alter zwischen 20 und 65 Jahren, im Jahr 2010 noch 34 so wird dieser bis 2060 auf 65 zunehmen und sich damit nahezu verdoppeln. Die größte Steigerung des Altenquotienten findet dabei allerdings bereits bis zum Jahr 2030 statt: Der Altenquotient wird dann etwa 50 betragen. Mit dem Anstieg des Altenquotienten geht ein Absinken des Erwerbspersonenpotentials im Alter von 20 bis unter 65 Jahren einher. Waren im Jahr 2009 noch 50 Millionen Menschen in dieser Altersgruppe, so werden es 2030 noch 44 Millionen sein. Im Jahr 2060 zählen zu jener Altersgruppe noch 35 Millionen Personen.

\footnotetext{
${ }^{3}$ Angesichts der in den nachfolgenden Abschnitten betrachteten Vielzahl an Erwerbs- und Arbeitszeitszenarien wird im vorliegenden Beitrag aus Gründen der Übersichtlichkeit lediglich ein mögliches Szenario zur Bevölkerungsentwicklung betrachtet. Dies stellt insbesondere im Hinblick auf die Rolle der Zuwanderung zur Sicherung des Arbeitskräftebedarfs eine Einschränkung dar.
}

\subsection{Erwerbsbeteiligung}

\subsubsection{Kohorten-Komponenten-Modell}

Zur Projektion der zukünftigen Erwerbsbeteiligung orientiert sich die vorliegende Arbeit an der Kohorten-Komponenten-Methode, die von Burniaux et al. (2004, S. 27ff.) eingeführt und von der Europäischen Kommission (2006, S. 175ff.) verfeinert wurde. Der Vorteil dieser Methodik gegenüber einer reinen Querschnittsbetrachtung, in der entweder die Querschnittstrends in die Zukunft fortgeschrieben oder aber der Querschnitt einfach konstant gehalten wird, liegt darin, dass die kohortenspezifische Dynamik aufgegriffen wird, und hierbei auch aktuelle Rechtsänderungen, die Auswirkungen auf das Erwerbsverhalten haben, berücksichtigt werden (Werding und Hofmann 2008, S. 21f.). EntryRates können dabei generell dann ermittelt werden, wenn der Anstieg der Erwerbsquote (EQ) für eine Alterskohorte positiv ist. Wie (1) zeigt, wird die Entry-Rate durch die Differenz der Erwerbsquote einer Alterskohorte im Zeitpunkt $t$ und $t-1$ ermittelt, die dann durch den Bevölkerungsanteil, der zum Zeitpunkt $t-1$ nicht in der Erwerbsbevölkerung vertreten aber erwerbsfähig ist, dividiert wird. ${ }^{4}$

$E N_{x, x+1}=\frac{E Q_{x+1, x+2}^{t}-E Q_{x, x+1}^{t-1}}{E Q_{\max }-E Q_{x, x+1}^{t-1}}$

\footnotetext{
${ }^{4} x=$ Alter; $t=$ Zeitpunkt. Bezüglich der maximal möglichen Erwerbsquote der Frauen unterscheiden sich die Annahmen von Burniaux et al. (2004, S. 43) und der Europäischen Kommission (2006, S. 176). So betrachten Erstgenannte eine Frauenerwerbsquote von 95 Prozent als Ma-
} 
Die Exit Rate berechnet sich für beide Geschlechter gemäß (2), wobei hier die Differenz der Erwerbsquote einer Alterskohorte in $t-1$ und $t$ in Bezug zur Erwerbsquote dieser Alterskohorte in $t-1$ gesetzt wird.

$E X_{x, x+1}=\frac{E Q_{x, x+1}^{t-1}-E Q_{x+1, x+2}^{t}}{E Q_{x, x+1}^{t-1}}$

Die Fortschreibung der Erwerbsquoten erfolgt dann gemäß (3) und (4):

$$
\begin{aligned}
& E Q_{x+1, x+2}^{t+1}=E Q_{x, x+1}^{t}+E N_{x, x+1}\left(E Q_{\max }-E Q_{x, x+1}^{t}\right), \\
& \quad \text { wenn } E N_{x, x+1}>0 \\
& E Q_{x+1, x+2}^{t+1}=\left(1-E X_{x, x+1}\right) E Q_{x, x+1}^{t}, \\
& \quad \text { wenn } E X_{x, x+1}>0
\end{aligned}
$$

\subsubsection{Datenbasis}

Als Ausgangspunkt liegen der vorliegenden Erwerbspersonenprojektion Daten des Mikrozensus zur Erwerbsbeteiligung nach Alter und Geschlecht aus dem Zeitraum 2005 bis 2010 zugrunde. $^{5}$ Dies ermöglicht eine alters- und geschlechtsspezifische Projektion der zukünftigen Erwerbsbeteiligung.

Um mögliche Einflüsse der Konjunktur auf die Erwerbsquoten zu glätten, wird ein geschlechts- und altersspezifischer 5-Jahres-Mittelwert berechnet. Die Ausgangsdaten zur Fortschreibung mittels des Kohorten-KomponentenModells bestehen somit aus 5-Jahres-Durchschnittsdaten der Jahre 2005 bis 2009 und 2006 bis 2010. Da die Zahl der Erwerbspersonen gemäß dem Mikrozensus nicht mit der Zahl der Erwerbspersonen gemäß der Volkswirtschaftlichen Gesamtrechnung (VGR) übereinstimmt, wurden die aus dem Mikrozensus gewonnenen Erwerbsquoten nach der konjunkturellen Glättung so korrigiert, dass die implizier-

ximum, wohingegen Letztgenannte dieses bei 99 Prozent sehen. Bezüglich der maximalen Erwerbsquote der Männer wird von beiden Studien ein Wert von 99 Prozent als Obergrenze angesehen. Vorliegende Arbeit orientiert sich an den Annahmen von (Burniaux et al. 2004), da die Autoren eine Frauenerwerbsquote von 99 Prozent mit Hinblick auf die schwangerschaftsbedingten Erwerbsunterbrechungen als unrealistisch einstufen. Wie sich im Verlauf der Studie jedoch gezeigt hat, ist die Sensitivität der Ergebnisse bezüglich dieser Annahme gering.

${ }^{5}$ Als erwerbstätig wird gemäß dem Labour Force Konzept der ILO jede Person angesehen, die zwischen 15 und 74 Jahren alt ist, in einem Arbeitsverhältnis mit mindestens einer Stunde Arbeitszeit je Woche steht oder selbstständig, Soldat/Zivildienstleistender, mithelfender Familienangehöriger oder Auszubildender ist. Als erwerbslos gelten all jene Personen, die zwischen 15 und 74 Jahren alt sind, kein Beschäftigungsverhältnis haben, Arbeit suchen und für eine Beschäftigung verfügbar sind. Die Erwerbstätigen und die Erwerbslosen bilden zusammen die Erwerbspersonen (Rengers 2004, S. 1374)

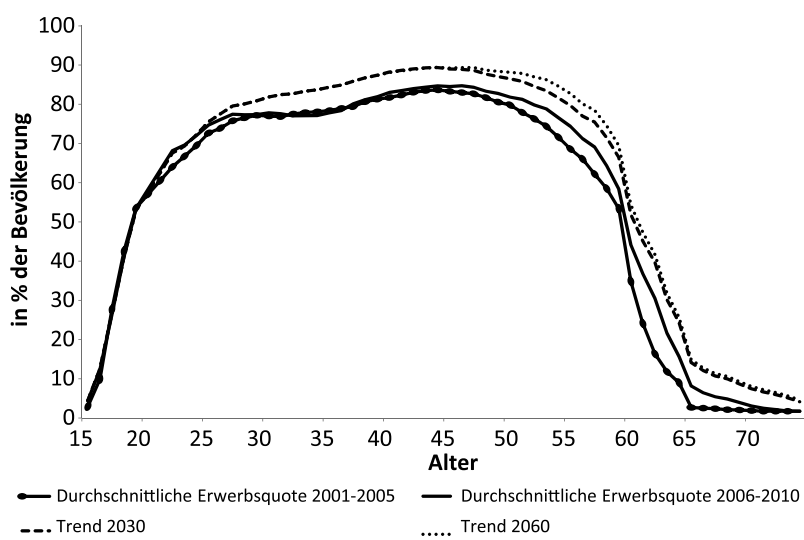

Abb. 1 Entwicklung der Erwerbsbeteiligung [Frauen] (Quelle: Mikrozensus, eigene Berechnungen)

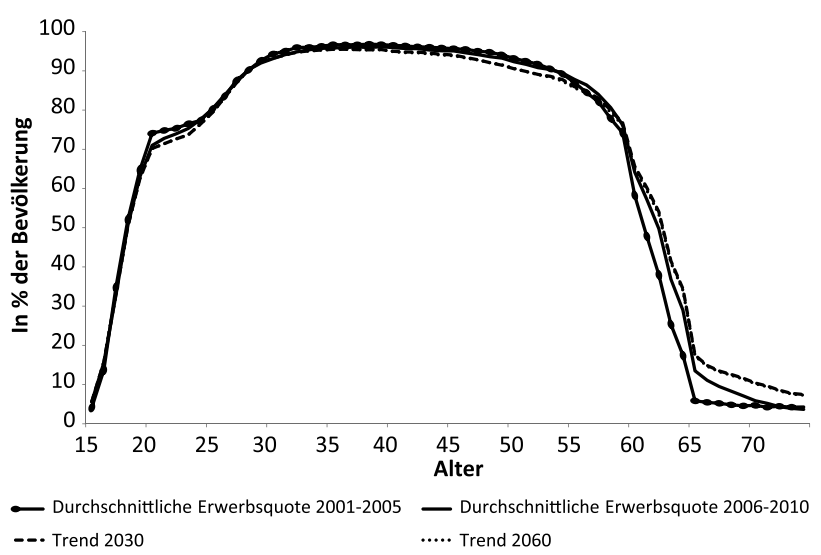

Abb. 2 Entwicklung der Erwerbsbeteiligung [Männer] (Quelle: Mikrozensus, eigene Berechnungen)

te Zahl der Erwerbspersonen derjenigen der VGR im Jahr 2010 entspricht. ${ }^{6}$

Die Abb. 1 und 2 zeigen die alters- geschlechtsspezifischen Erwerbsquoten im Durchschnitt der Jahre 2001 bis

${ }^{6}$ Die Skalierung wird im Anschluss an die jeweilige Erwerbsquotenprojektion vorgenommen und erfolgt additiv und gleichmässig auf alle Altersjahre verteilt.

Wie Fuchs and Söhnlein (2003) zeigen, besteht beim Mikrozensus zudem das strukturelle Problem der Untererfassung geringfügig Beschäftigter, wobei sich diese Untererfassung vor allem bei den 15 - bis 19-Jährigen und über 59-Jährigen häuft. Eine Korrektur dieser Untererfassung ist auf Grundlage von Fuchs and Söhnlein (2003) möglich und wurde bei den ersten Berechnungen für die vorliegende Arbeit berücksichtigt. Im weiteren Verlauf der Studie wurde diese Korrektur jedoch wieder verworfen. Hauptgrund dafür waren unrealistisch hohe Erwerbsquoten für die über 64-Jährigen, die durch die Kombination des Kohorten-Komponenten-Modells mit der Korrektur entstanden. So ergab eine Projektion der männlichen Erwerbsquote auf Basis des in Abschn. 2.2.1 vorgestellten Kohorten-Komponenten-Modells Werte von ca. 20 Prozent für über 70-Jährige in 2030. Dies ist vergleichbar mit den Erwerbsquoten von Estland oder Lettland heute, die eine Altersarmut von 40 Prozent und mehr aufweisen (Antuofermo and Meglio 2012, S. 2). 


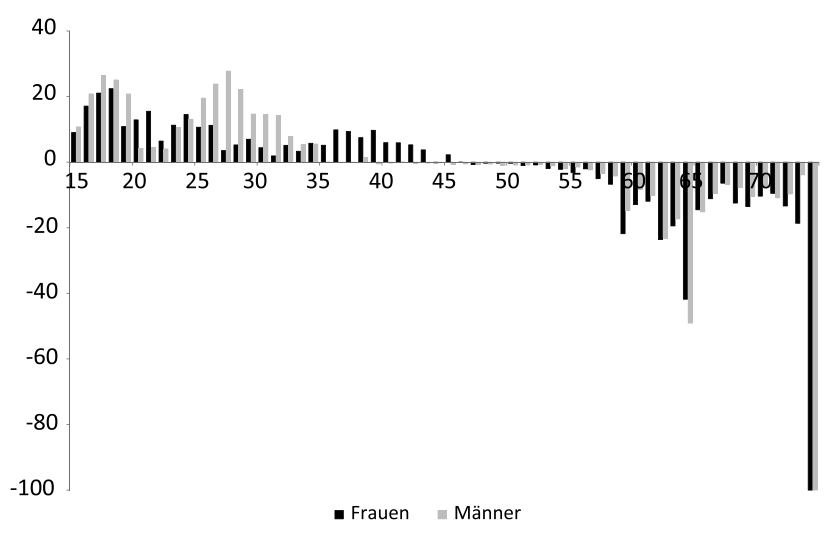

Abb. 3 Entry- und Exit-Rates von Frauen und Männern (Quelle: Mikrozensus, eigene Berechnungen)

2005 bzw. 2006 bis 2010. Deutlich zu erkennen ist dabei die Dynamik, mit welcher sich die Erwerbsbeteiligung in den vergangenen 10 Jahren entwickelt hat. Diese ist bei den Frauen dabei deutlich stärker ausgeprägt als bei den Männern. Dieser Unterschied ist unter anderem auf das Rentenreformgesetz von 1992 zurückzuführen, als Folge dessen das abschlagsfreie Renteneintrittsalter für Frauen und langjährig Versicherte angehoben sowie versicherungsmathematische Abschläge eingeführt wurden (Deutsche Rentenversicherung 2011, S. 289f). ${ }^{7}$ Da die Umsetzung dieser Reform bei den Frauen erst zwischen den Jahren 2000 und 2009 stattfand, während sie bei den langjährig Versicherten im Zeitraum zwischen 2000 und 2003 erfolgte, lässt sich ein Teil der (größeren) weiblichen Dynamik hiermit erklären. Nicht zuletzt dürfte aber auch die allgemein steigende Erwerbsbeteiligung der Frauen, die sich seit den 1970er Jahren zeigt, hierzu beigetragen haben.

In Abb. 3 sind die auf Basis des Mikrozensus ermittelten Entry- und Exit-Rates für Männer und Frauen dargestellt. ${ }^{8}$ Beginnend mit dem 15. Lebensjahr steigen die Entry-Rates für beide Geschlechter bis zum 19. Lebensjahr an, wobei der Anstieg der Männer unter anderem bedingt durch die Wehrpflicht durchgängig stärker ausfällt. Das Abfallen der Entry-Rates ab dem 19. Lebensjahr deutet darauf hin, dass zu diesem Zeitpunkt keine nennenswerte Erhöhung der Erwerbsbeteiligung mehr stattfindet, also all jene Personen, die sich für eine Ausbildung entschieden haben, im Arbeitsmarkt vertreten sind, wohingegen das übrige Erwerbspersonenpotential sich in einer Ausbildung befindet, die keine Zurechnung zu den Erwerbspersonen erlaubt. Bis zum 40.

\footnotetext{
${ }^{7}$ Für langjährig Versicherte wurde dabei das abschlagsfreie Rentenzugangsalter von 63 auf 65 Jahre erhöht, während für Frauen das abschlagsfreie Rentenzugangsalter von 60 auf 65 Jahre angehoben wurde. Ein früherer Rentenzugang wird seit der Anhebung der Altersgrenzen mit einem Rentenabschlag von 0,3 Prozent pro Monat abgegolten.

${ }^{8}$ Die Entry- und Exit-Rates werden auf Basis der Durchschnittsdaten 2005-2009 und 2006-2010 berechnet.
}

Lebensjahr steigen die Entry-Rates für beide Geschlechter wieder an, wobei die Entry-Rates der Männer ihr Maximum im Alter von 27 Jahren erreichen. Der im Vergleich flachere Verlauf der weiblichen Entry-Rates lässt sich im wesentlichen auf Auszeiten in Zusammenhang mit Schwangerschaften zurückführen. Ab dem 45. Lebensjahr wandeln sich die Entry-Rates dann in Exit-Rates um, wobei deren Höhe mit zunehmendem Alter ansteigt und für beide Geschlechter im Alter von 64 Jahren ihr Maximum erreicht. Annahmegemäß beträgt die Erwerbsquote im Alter von 75 Jahren Null, sodass die Exit-Rates im Alter von 74 Jahren den Wert 100 erreichen.

\section{Erwerbspersonenszenarien im Vergleich}

Im Weiteren werden zehn verschiedene Erwerbsquotenszenarien betrachtet, deren Annahmen in Abschn. 3.1 erläutert werden. Aufbauend darauf werden die Ergebnisse dieser Szenarien in Abschn. 3.2 diskutiert.

\subsection{Alternativszenarien zur Entwicklung der Erwerbsbeteiligung}

\section{Szenario 1: Status Quo}

Um den Vergleich zu den Erwerbsquoten des Status Quo zu ermöglichen, werden in diesem Szenario die durchschnittlichen Erwerbsquoten der Jahre 2006 bis 2010, die in Abb. 1 und 2 dargestellt sind, konstant gehalten.

\section{Szenario 2: Trend}

Szenario 2 entspricht dem Kohorten-Komponenten-Modell, dass in Abschn. 2.2.1 vorgestellt wurde. Abbildung 1 zeigt dabei die projizierten Erwerbsquoten der Frauen für die Jahre 2030 und 2060, wobei hier zu erkennen ist, dass sich die Erwerbsquoten für fast alle Altersjahre erhöhen. $\mathrm{Zu}$ beachten ist hierbei, dass noch keine Annahme bezüglich der Auswirkungen der Rente mit 67 getroffen wurde, sich die Verschiebung also rein aus der kohortenspezifischen Fortschreibung ergibt. ${ }^{9}$ Die projizierten Erwerbsquoten der Männer in Abb. 2 unterscheiden sich insoweit von denen der Frauen, als dass sich eine Erhöhung der Erwerbsquote erst ab dem 59. Lebensjahr abzeichnet. Der ab dem 39. Lebensjahr zu beobachtende Abnahmetrend der männlichen Erwerbsquote lässt sich durch die positiven Exit-Rates in Abb. 3 begründen, wobei die Ursachen hierfür nicht abschließend geklärt werden konnten. Wie auch bei den Frauen fallen die Erwerbsquoten der Jüngeren im Alter zwischen 18 und 25 Jahren leicht ab, was nach Einschätzung der Autoren durch

\footnotetext{
${ }^{9}$ Da die schrittweise Einführung der Rente mit 67 erst in den Jahren 2012 bis 2029 erfolgt, zeigen sich ihre Auswirkungen erst in der $\mathrm{Zu}$ kunft in den Daten des Mikrozensus.
} 
den anhaltenden Trend zur Höherqualifizierung erklärt werden kann.

\section{Szenario 3 (Basisszenario): Rente mit 67}

Da das vorliegende Projektionsmodell aus Szenario 2 die Auswirkungen der Rente mit 67 nicht in die Berechnungen mit einbezieht, wird in Szenario 3 die Annahme getroffen, dass die Rente mit 67 das effektive Renteneintrittsalter um ein Jahr nach hinten verschiebt. Die Berechnung des effektiven Renteneintrittsalter findet dabei aus den Erwerbsquoten heraus statt und ist deshalb nicht mit dem effektiven Renteneintrittsalter der Deutschen Rentenversicherung identisch. Es ähnelt vielmehr einem Erwerbsaustrittsalter und wird im Querschnitt mittels (5) berechnet (Keese 2007, S. 1f; Scherer 2002, S. 14).

$A R_{t}=\frac{\sum_{a=41}^{75} a\left(E Q_{t}^{a-1}-E Q_{t}^{a}\right)}{E Q_{t}^{40}}$

Entsprechend der in (5) zugrunde gelegten Definition steigt das effektive Renteneintrittsalter für Frauen (Männer) von $61,2(62,1)$ Jahren in 2010 allein infolge der kohortenspezifischen Trendfortschreibung aus Szenario 2 auf 62,1 $(62,7)$ Jahre in 2030 an. Die Anpassung bezüglich der Rente mit 67 erfolgt nun zusätzlich zu diesem Anstieg und verschiebt das effektive Renteneintrittsalter um ein weiteres Jahr nach hinten. Die Abb. 4 und 5 zeigen die neuen Erwerbsquotenprofile beispielhaft für das Jahr 2030. Im weiteren Verlauf der Arbeit stellt dieses Szenario das Basisszenario dar und ist somit Grundlage für jene Altersgruppen, die in den Szenarien 5 bis 8 keiner Änderung unterliegen.

\section{Szenario 4: Renteneintritt - Variante a}

Da letztlich nicht genau vorhergesagt werden kann, inwieweit die Rente mit 67 das effektive Erwerbsaustrittsalter verschiebt, wird in diesem Szenario ein Anstieg des effektiven Renteneintrittsalters von zusätzlich zwei Jahren modelliert. Das effektive Renteneintrittsalter liegt für Frauen (Männer) im Jahr 2030 dann bei 64,1 $(64,7)$ Jahren. Abbildung 4 und 5 zeigen die so gewonnenen Erwerbsquotenprofile im Jahr 2030 .

\section{Szenario 5: Erwerbseintritt}

Durch die Einführung des achtjährigen Gymnasiums, den Übergang zu Bachelorabschlüssen im Zuge des BolognaProzesses und durch die Abschaffung der Wehrpflicht dürfte das Erwerbseintrittsalter in den kommenden Jahren weiter abnehmen. Um die Auswirkungen dieser Entwicklung für die Zahl der Erwerbspersonen abzuschätzen, unterstellt das Szenario „Erwerbseintritt“, dass das durchschnittliche Erwerbseintrittsalter bis 2020 um ein Jahr absinkt. Ferner wurde unterstellt, dass die angesprochenen Entwicklungen sich lediglich in der Altersgruppe der 20- bis 29-Jährigen in einem

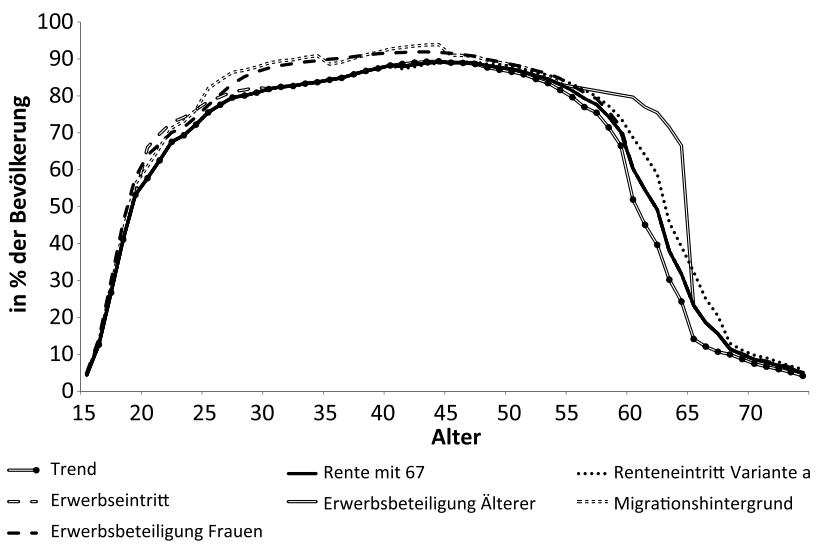

Abb. 4 Erwerbsbeteiligung in den Szenarien 2 bis 8 im Jahr 2030 [Frauen] (Quelle: Mikrozensus, eigene Berechnungen)

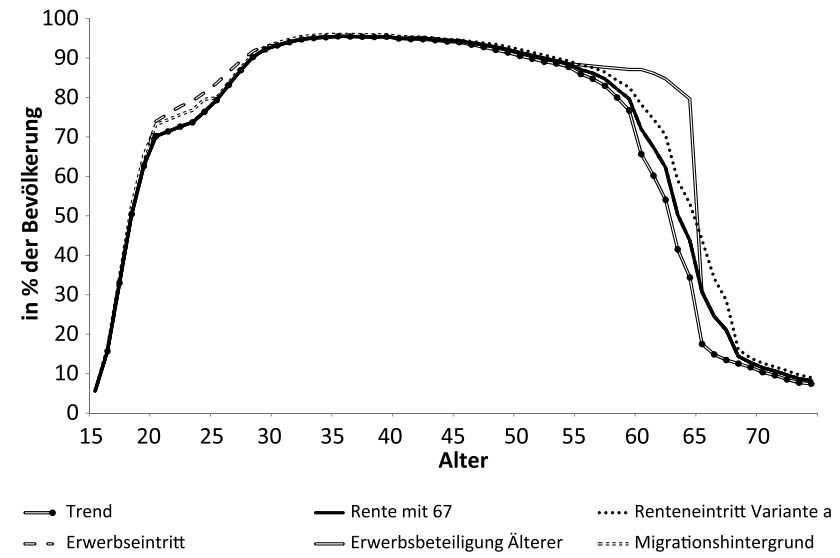

Abb. 5 Erwerbsbeteiligung in den Szenarien 2 bis 8 im Jahr 2030 [Männer] (Quelle: Mikrozensus, eigene Berechnungen)

früheren Erwerbseintritt niederschlagen. ${ }^{10}$ Die Abb. 4 und 5 zeigen die neu gewonnenen Erwerbsquotenprofile im Jahr 2030.

\section{Szenario 6: Erwerbsbeteiligung Älterer}

Eine höhere Erwerbsbeteiligung Älterer wird nicht zuletzt von der Bundesregierung als ein Handlungsfeld zur Sicherung des Arbeitskräftepotentials angesehen. Vor diesem Hintergrund quantifiziert das Szenario „Erwerbsbeteiligung Älterer“ die Auswirkungen einer erhöhten Erwerbsbeteiligung in der Altersgruppe der 60- bis unter 65-Jährigen für die Entwicklung der Erwerbspersonenzahl. Konkret wird dabei unterstellt, dass sich die Erwerbsquoten der 60- bis unter 65-Jährigen bis zum Jahr 2030 linear an das Niveau

\footnotetext{
${ }^{10}$ Das Erwerbseintrittsalter wird hierbei als gewichtetes arithmetisches Mittel berechnet: $E A=\sum_{a=20}^{29} a *$ Gewicht $_{a}$, wobei Gewicht ${ }_{a}=$ $\frac{E Q_{t}^{a}-E Q_{t}^{a-1}}{\sum_{a=20}^{29}\left(E Q_{t}^{a}-E Q_{t}^{a-1}\right)}$. Ausgehend von einem Erwerbseintrittsalter der Frauen (Männer) von 22,5 (24) Jahren in 2010, sinkt dieses im Szenario „Erwerbseintritt“ langfristig auf $22(23,2)$ Jahre ab.
} 
der 55- bis unter 60-Jährigen aus Szenario 2 angleichen. Die neu gewonnenen Erwerbsquotenprofile im Jahr 2030 sind in Abb. 4 und 5 dargestellt.

\section{Szenario 7: Migrationshintergrund}

Die bessere Integration von Menschen mit Migrationshintergrund in den Arbeitsmarkt stellt für die Bundesregierung (2011, S. 109) ein zentrales Anliegen dar, ist sie doch ein wesentlicher Indikator für das Gelingen von Integration im Allgemeinen. Darüber hinaus verbirgt sich hinter diesem Anliegen ein weiteres Handlungsfeld zur Sicherung des Arbeitskräftepotentials. ${ }^{11}$ Die Auswirkungen einer höheren Erwerbsbeteiligung von Personen mit Migrationshintergrund werden daher im Szenario „Migrationshintergrund“ untersucht. Zusätzlich zur Trendfortschreibung der Erwerbsquoten wurde dabei unterstellt, dass sich die Erwerbsquoten von Personen mit Migrationshintergrund bis zum Jahr 2030 an diejenigen von Personen ohne Migrationshintergrund angleichen. Die Angleichung erfolgt dabei linear über den Anpassungszeitraum 2010 bis 2030. Wie Abb. 4 zeigt, existiert vor allem bei den Frauen im Alter von 20 bis 55 Jahren ein Steigerungspotential. Bei den Männern resultiert hingegen kaum noch eine Anhebung der Erwerbsquote (vgl. Abb. 5).

\section{Szenario 8: Erwerbsbeteiligung Frauen}

Wie in Abschn. 2.2.2 ausgeführt wurde, stieg vor allem die Erwerbsbeteiligung der Frauen in den letzten Jahren und Jahrzehnten stark an. Gründe hierfür sind unter anderem in den besseren Rahmenbedingungen zur Vereinbarkeit von Familie und Beruf, aber auch in einem höheren Anteil kinderloser Frauen zu suchen (Werding and Hofmann 2008, S. 24). Die Frage, welches Potential mit einer über das Basisszenario hinausgehenden weiblichen Erwerbsbeteiligung erschlossen werden kann, wird in Szenario 8 beantwortet. Es wird hierbei angenommen, dass sich der Abstand zwischen weiblicher und männlicher Erwerbsquote bis ins Jahr 2030 halbiert und dann konstant bleibt. ${ }^{12}$ Abbildung 4 zeigt die neuen Erwerbsquoten der Frauen im Vergleich zum Basisszenario, wobei auffällt, dass sich vor allem die Erwerbsquoten der 20- bis 55-Jährigen nach oben verschieben. Die Erwerbsquote der Männer, die von dieser Anpassung nicht betroffen ist, entwickelt sich gemäß dem Basisszenario.

\footnotetext{
${ }^{11}$ Vorliegender Arbeit liegt die Definition des Statistischen Bundesamt (2011a, S. 6) zur Migration im engeren Sinne zu Grunde: ,Zu den Menschen mit Migrationshintergrund zählen alle nach 1949 auf das heutige Gebiet der BRD zugewanderten, sowie alle in Deutschland geborenen Ausländer und alle in Deutschland als Deutsche Geborenen mit zumindest einem zugewanderten oder als Ausländer in Deutschland geborenen Elternteil." Dieser Definition folgend lebten in 2010 ca. 15,7 Millionen Menschen mit Migrationshintergrund in Deutschland (Statistisches Bundesamt 2011a, S. 34).

${ }^{12}$ Als Zielerwerbsquote der Männer in 2030 wird jene aus Szenario 2 herangezogen.
}

\section{Szenario 9: Kombinationsvariante}

In den Szenarien 4 bis 8 wird jeweils das Potential eines einzelnen Handlungsfeldes zur Sicherung des Arbeitskräftepotentials untersucht. Obwohl dies für sich genommen von Interesse ist, um die Hebelwirkung der einzelnen Handlungsfelder zu bemessen, wird eine effektive Strategie zur Sicherung des Arbeitskräftepotentials gleichzeitig an mehreren Handlungsfeldern ansetzen müssen. Im Hinblick auf die politische Zielsetzung ist dabei insbesondere von Interesse, welcher Effekt sich durch eine Kombination der hier betrachteten Handlungsfelder insgesamt erzielen lässt. Zu diesem Zweck werden im abschließenden Szenario 9 die Auswirkungen auf die Erwerbspersonenzahl untersucht, welche sich aus einem umfassenden Maßnahmenbündel ergeben. Auch ist eine Gesamtbetrachtung deshalb von Bedeutung, weil sich aufgrund von „Überschneidungen“ in der Summe ein geringerer Gesamteffekt ergibt als bei einer Summierung der jeweiligen Einzeleffekte. Im Einzelnen umfasst die Kombinationsvariante die Auswirkungen eines um 2 Jahre späteren Renteneintritts (Szenario 4), eines früheren Erwerbseintritts (Szenario 5), einer erhöhten Erwerbsbeteiligung Älterer (Szenario 6), einer erhöhten Erwerbsbeteiligung von Menschen mit Migrationshintergrund (Szenario 7) sowie einer Angleichung der Erwerbsbeteiligung zwischen den Geschlechtern (Szenario 8).

\subsection{Die zukünftige Zahl der Erwerbspersonen}

In Tab. 2 sind die Implikationen der einzelnen Szenarien für die Entwicklung der Erwerbspersonen im Zeitraum 2010 bis 2060 dargestellt. Im Sinne des maximalen Erwerbspotentials findet sich in Tab. 2 zum Vergleich auch die Entwicklung der Erwerbsbevölkerung entsprechend des in Abschn. 2.1 dargestellten und für die Berechnungen zugrunde gelegten demografischen Referenzszenarios. Betrachtet man zunächst den Fall, dass die Erwerbsbeteiligung in der Zukunft auf dem heutigen Niveau verharrt (Szenario 1, Status Quo), so ist aus Tab. 2 ersichtlich, dass in diesem Fall mit einem Rückgang der Erwerbspersonenzahl von aktuell 43 Millionen Personen auf 37 Millionen Personen in 2030 und 30 Millionen Personen in 2060 zu rechnen ist. Im Vergleich zu 2010 entspricht dies einem Rückgang um 15 Prozent bis 2030 bzw. 31 Prozent bis 2060. Mit anderen Worten fällt der Rückgang der Erwerbspersonen im Vergleich zur Erwerbsbevölkerung stärker aus, was im Wesentlichen auf die geringere Erwerbsquote der Älteren zurückzuführen ist. Allerdings erscheint ein solches Szenario wenig plausibel. So ist allein unter Fortschreibung der in den Daten zu beobachtenden kohortenspezifischen Trends zukünftig von einer Zunahme der Erwerbsbeteiligung der Frauen und der Älteren auszugehen (siehe hierzu Abschn. 2.2.2). Zwar sind diese kohorten- und geschlechtsspezifischen Trends (Szenario 2, Trend) bei Weitem nicht ausreichend, um den demo- 
Tab. 2 Übersicht der vorausberechneten Erwerbspersonenzahl

\begin{tabular}{|c|c|c|c|c|c|c|}
\hline & \multicolumn{6}{|c|}{ Personen in Tausend } \\
\hline & 2010 & 2020 & 2030 & 2040 & 2050 & 2060 \\
\hline $\begin{array}{l}\text { Erwerbspersonenpotential: Bevölkerung } \\
\text { im Alter von } 15 \text { bis } 74 \text { Jahren }\end{array}$ & 63801 & 60577 & 58266 & 53613 & 49574 & 46909 \\
\hline \multirow[t]{2}{*}{ Szenario 1: Status Quo } & 43298 & 40945 & 36824 & 34299 & 32131 & 29918 \\
\hline & $(0)$ & $-(1696)$ & $-(2650)$ & $-(2302)$ & $-(2293)$ & $-(2147)$ \\
\hline \multirow[t]{2}{*}{ Szenario 2: Trend } & 43298 & 42140 & 38359 & 35755 & 33546 & 31261 \\
\hline & $(0)$ & $-(501)$ & $-(1114)$ & $-(847)$ & $-(878)$ & $-(804)$ \\
\hline \multirow[t]{2}{*}{ Basisszenario 3: Rente mit 67} & 43298 & 42642 & 39474 & 36602 & 34424 & 32065 \\
\hline & $(0)$ & $(0)$ & $(0)$ & $(0)$ & $(0)$ & $(0)$ \\
\hline \multirow[t]{2}{*}{ Szenario 4: Renteneintritt - Variante a } & 43298 & 43143 & 40588 & 37449 & 35302 & 32869 \\
\hline & $(0)$ & $(501)$ & (1114) & $(847)$ & $(878)$ & $(804)$ \\
\hline \multirow[t]{2}{*}{ Szenario 5: Erwerbseintritt } & 43298 & 42955 & 39757 & 36878 & 34685 & 32302 \\
\hline & $(0)$ & $(313)$ & $(283)$ & $(276)$ & $(262)$ & $(237)$ \\
\hline \multirow[t]{2}{*}{ Szenario 6: Erwerbsbeteiligung Älterer } & 43298 & 43268 & 41307 & 37848 & 35763 & 33256 \\
\hline & $(0)$ & $(626)$ & (1833) & $(1246)$ & (1339) & $(1191)$ \\
\hline \multirow[t]{2}{*}{ Szenario 7: Migrationshintergrund } & 43298 & 43081 & 40282 & 37365 & 35141 & 32738 \\
\hline & $(0)$ & $(440)$ & $(808)$ & $(763)$ & (717) & $(673)$ \\
\hline \multirow[t]{2}{*}{ Szenario 8: Erwerbsbeteiligung Frauen } & 43298 & 42657 & 40120 & 37036 & 34774 & 32398 \\
\hline & $(0)$ & $(15)$ & $(646)$ & $(434)$ & $(350)$ & (333) \\
\hline \multirow[t]{2}{*}{ Szenario 9: Kombinationsvariante } & 43298 & 44149 & 42726 & 39133 & 36988 & 34410 \\
\hline & $(0)$ & $(1507)$ & $(3253)$ & $(2531)$ & $(2564)$ & $(2345)$ \\
\hline
\end{tabular}

Quelle: Eigene Berechnungen; Abweichungen vom Basisszenario in Klammern

grafisch bedingten Rückgang der Erwerbspersonenzahl auszugleichen. Im Vergleich zu einer reinen Fortschreibung der heutigen Erwerbsquoten fällt der Rückgang der Erwerbspersonenzahl mit 11 Prozent bis 2030 bzw. 28 Prozent bis 2060 jedoch spürbar geringer aus. Ein weiterer positiver Effekt auf die Erwerbsbeteiligung ist schließlich von der Einführung der Rente mit $67 \mathrm{zu}$ erwarten. Unterstellt man, dass sich neben der Zunahme der Erwerbsbeteiligung entsprechend der kohortenspezifischen Trends langfristig auch das effektive Rentenzugangsalter infolge der Rente mit 67 um $1 \mathrm{Jahr}$ erhöht (Szenario 3, Basisszenario), so ergibt sich ein Rückgang der Erwerbspersonen auf 39 Millionen Personen in 2030 bzw. 32 Millionen Personen in 2060. Im Vergleich zu 2010 entspricht dies einem Rückgang um 9 Prozent bis 2030 bzw. 26 Prozent bis 2060. Trotz ihres dämpfenden Effekts bleibt damit festzuhalten, dass zukünftige Verhaltensänderungen (kohorten- und geschlechtsspezifische Trends) und bereits beschlossene Reformmaßnahmen (Rente mit 67) lediglich ausreichend sind, um die Erwerbsbeteiligung bis zum Jahr 2020 in etwa auf dem heutigen Niveau zu stabilisieren. Dies spiegelt sich deutlich in der Entwicklung der (gesamtwirtschaftlichen) Erwerbsquote (Anteil der Erwerbspersonen an der Gesamtbevölkerung) wider (vgl. Tab. 3). Während bis 2020 eine annähernde Stabilisierung der Erwerbsquote auf dem heutigen Niveau von 53 Pro- zent gelingt, sinkt diese trotz der Zunahme der Erwerbsbeteiligung der Älteren und der Frauen sowie der Rente mit 67 im Basisszenario bis 2030 um etwa 3 Prozentpunkte ab.

Um den demografisch bedingten Rückgang des Arbeitskräfteangebots zu kompensieren, werden daher zukünftig noch weitere Maßnahmen notwendig sein. Aufbauend auf dem Basisszenario soll entsprechend der in Abschn. 3.1 formulierten Alternativszenarien nachfolgend aufgezeigt werden, welche Handlungsfelder hierzu am ehesten geeignet erscheinen. Da der Anteil der über 50-Jährigen infolge des demografischen Altersstruktureffekts bis ins Jahr 2030 zunehmen wird, birgt jede Strategie, die die Erwerbsbeteiligung dieser Altersgruppe weiter erhöht, das Potential in sich, dem Rückgang der Erwerbspersonenzahl effektiv entgegen zu wirken. So erhöht jede weitere Verschiebung des effektiven Rentenzugangsalters um ein weiteres Jahr nach hinten die Zahl der Erwerbspersonen, wobei die größten Effekte bis ins Jahr 2030 hinein zu erzielen sind (Szenario 4, Renteneintritt Variante a). Ein Umstand der letztlich der demografischen Entwicklung geschuldet ist. Wie Tab. 3 dabei ebenfalls zeigt, geht die Erhöhung des Rentenzugangsalter um ein weiteres Jahr (gegenüber dem Basisszenario) mit einer Erhöhung des Anteils der Erwerbspersonen an der Gesamtbevölkerung um durchschnittlich 1,1 Prozentpunkte einher. Hingegen ergibt sich am anderen Ende des Altersspektrums 
Tab. 3 Anteil der Erwerbspersonen an der Gesamtbevölkerung

\begin{tabular}{|c|c|c|c|c|c|c|}
\hline & \multicolumn{6}{|c|}{ Angaben in Prozent der Gesamtbevölkerung } \\
\hline & 2010 & 2020 & 2030 & 2040 & 2050 & 2060 \\
\hline \multirow[t]{2}{*}{ Szenario 1: Status Quo } & 52.9 & 50.9 & 46.9 & 45.3 & 44.4 & 43.6 \\
\hline & $(0)$ & $-(2.1)$ & $-(3.4)$ & $-(3)$. & $-(3.2)$ & $-(3.1)$ \\
\hline \multirow[t]{2}{*}{ Szenario 2: Trend } & 52.9 & 52.4 & 48.9 & 47.2 & 46.4 & 45.6 \\
\hline & $(0)$ & $-(0.6)$ & $-(1.4)$ & $-(1.1)$ & $-(1.2)$ & $-(1.2)$ \\
\hline \multirow[t]{2}{*}{ Basisszenario 3: Rente mit 67} & 52.9 & 53.1 & 50.3 & 48.3 & 47.6 & 46.8 \\
\hline & $(0)$ & (0) & $(0)$ & $(0)$ & $(0)$ & (0) \\
\hline \multirow[t]{2}{*}{ Szenario 4: Renteneintritt - Variante a } & 52.9 & 53.7 & 51.7 & 49.4 & 48.8 & 48.0 \\
\hline & $(0)$ & $(0.6)$ & $(1.4)$ & $(1.1)$ & $(1.2)$ & $(1.2)$ \\
\hline \multirow[t]{2}{*}{ Szenario 5: Erwerbseintritt } & 52.9 & 53.4 & 50.6 & 48.7 & 47.9 & 47.1 \\
\hline & $(0)$ & $(0.4)$ & $(0.4)$ & $(0.4)$ & $(0.4)$ & $(0.3)$ \\
\hline \multirow[t]{2}{*}{ Szenario 6: Erwerbsbeteiligung Älterer } & 52.9 & 53.8 & 52.6 & 50.0 & 49.4 & 48.5 \\
\hline & $(0)$. & $(0.8)$ & (2.3) & (1.6) & (1.9) & $(1.7)$ \\
\hline \multirow[t]{2}{*}{ Szenario 7: Migrationshintergrund } & 52.9 & 53.6 & 51.3 & 49.3 & 48.6 & 47.8 \\
\hline & $(0)$ & $(0.5)$ & (1.) & (1.) & (1.) & (1.) \\
\hline \multirow[t]{2}{*}{ Szenario 8: Erwerbsbeteiligung Frauen } & 52.9 & 53.1 & 51.1 & 48.9 & 48.1 & 47.3 \\
\hline & $(0)$ & $(0)$. & $(0.8)$ & $(0.6)$ & $(0.5)$ & $(0.5)$ \\
\hline \multirow[t]{2}{*}{ Szenario 9: Kombinationsvariante } & 52.9 & 54.9 & 54.4 & 51.7 & 51.1 & 50.2 \\
\hline & $(0)$ & (1.9) & $(4.1)$ & $(3.3)$ & (3.5) & $(3.4)$ \\
\hline
\end{tabular}

Quelle: Eigene Berechnungen, Abweichungen vom Basisszenario in Klammern

nur ein vergleichsweise geringer Effekt. So summieren sich die Auswirkungen eines um ein Jahr vorgezogenen Erwerbseintritts (Szenario 5, Erwerbseintritt) auf durchschnittlich 274.000 Erwerbspersonen, was einer Steigerung des Anteils der Erwerbspersonen gegenüber dem Basisszenario von 0,4 Prozentpunkten entspricht (vgl. Tab. 3). Hingegen sind die Auswirkungen einer Anhebung der Erwerbsquote der 60bis unter 65-Jährigen auf die der 55- bis unter 60-Jährigen (Szenario 6, Erwerbsbeteiligung Älterer) stärker als bei denen einer Anhebung des effektiven Renteneintrittsalters um ein Jahr und summieren sich im Schnitt auf einen Zugewinn von ca. 1,2 Millionen Erwerbspersonen zusätzlich zum Basisszenario. Der Effekt in Tab. 3 zeigt dabei, dass der Hebel dieses einzelnen Politikszenarios nicht nur am größten sondern dieses Szenario bereits allein ausreichend ist, um die die gesamtwirtschaftliche Erwerbsquote bis zum Jahr 2030 auf dem aktuellen Niveau zu stabilisieren. Die Angleichung der Erwerbsquote von Menschen mit Migrationshintergrund (Szenario 7, Migrationshintergrund) hat zur Folge, dass sich ihr Ergebnis zwischen dem eines früheren Erwerbseintritts und dem einer Anhebung der Erwerbsbeteiligung Älterer findet, und somit in etwa dem des Szenarios „Renteneintritt - Variante a“ entspricht. Die Halbierung des Abstands der Erwerbsquote von Frauen und Männern (Szenario 8, Erwerbsbeteiligung Frauen) wirkt sich, wie aus Tab. 2 und $3 \mathrm{zu}$ sehen ist, erst ab dem Jahr 2030 aus, was unter anderem darauf zurückzuführen ist, dass die Erwerbsquo- te der Frauen bis 2030 allein durch die kohortenspezifische Fortschreibung stark ansteigt. Das Maximalszenario (Szenario 9, Kombinationsvariante) stellt schließlich den kumulierten Effekt bei gleichzeitiger Umsetzung eines umfassenden Maßnahmenbündels dar. Im Vergleich zum Jahr 2010 kann die Erwerbsquote durch die hier betrachtete Kombination mehrerer Szenarien bis zum Jahr 2030 um 1,5 Prozentpunkte gesteigert werden (vgl. Tab. 3). Nach 2030 sinken die Erwerbspersonenzahl und die Erwerbsquote jedoch auch in diesem Maximalszenario schnell und deutlich ab, was einerseits auf das fallende Erwerbspersonenpotential zurückzuführen ist. Andererseits ist das Absinken der Erwerbspersonenzahl jedoch auch darauf zurückzuführen, dass die hier betrachteten Szenarien im Zeitraum nach 2030 keine weiteren Anpassungen der Erwerbsbeteiligung vorsehen. ${ }^{13}$

\section{Der Effekt eines steigenden Arbeitsvolumens}

Die bisherige Betrachtung der Erwerbspersonen beschränkte sich allein auf die zukünftige Entwicklung des extensiven Arbeitsangebots, also die Anzahl der am Arbeitsmarkt aktiven Personen. Nicht berücksichtigt wurde hingegen die

\footnotetext{
${ }^{13}$ So wäre es durchaus möglich, dass sich nach 2030 eine über das Szenario 8 hinausgehende Angleichung der Frauenerwerbsquote an die Männererwerbsquote ergibt.
} 
Frage des intensiven Arbeitsangebots, also die Entwicklung der von den Erwerbspersonen geleisteten Arbeitsstunden. Insofern lieferte die bisherige Betrachtung nur ein grobes Bild der zukünftigen Entwicklung des Arbeitsangebots. Aufbauend auf dem in Abschn. 3.1 betrachteten Basisszenario zur zukünftigen Entwicklung der Erwerbsbeteiligung sollen daher nachfolgend zunächst die Implikationen für die Entwicklung des Arbeitsvolumens, d.h. der geleisteten Arbeitsstunden, betrachtet werden. Daran anknüpfend wird anhand verschiedener Szenarien untersucht, in welchem Umfang eine Erhöhung des Jahresarbeitszeitvolumens je Erwerbstätigen einen Beitrag zur Abfederung der demografisch bedingten Schrumpfung der Erwerbspersonenzahl leisten kann. Die einzelnen Szenarien unterscheiden sich dabei im Hinblick auf die unterstellte Erwerbslosenquote, die Teilzeitquote sowie die Arbeitszeit je Teilzeitbeschäftigten. Abschließend werden diese einzelnen Jahresarbeitszeitszenarien dann auf die implizierte Entwicklung des realen Bruttoinlandsprodukts je Einwohner untersucht, und es wird so gezeigt, welche jährlichen Produktivitätszuwächse nötig sind, um das Bruttoinlandsprodukt je Einwohner auch in Zukunft konstant zu halten.

\section{Arbeitszeitszenario 1: Status Quo}

Als Ausgangspunkt unterstellt das Szenario „Status Quo“, dass die (alters- und geschlechtsspezifischen) Erwerbslosenquoten, die Teilzeitquoten sowie die normalerweise geleistete Arbeitszeit je Vollzeit-/Teilzeitbeschäftigen jeweils dem Niveau des Jahres 2010 entsprechen. ${ }^{14}$ Diese Szenario dient als Ausgangsszenario für die nachfolgenden vier Modellrechungen.

\section{Arbeitszeitszenario 2: Halbierung der Erwerbslosen- quote}

Bei einer einfachen Angebots- und Nachfragebetrachtung des Arbeitsmarktes wird der demografisch bedingte Rückgang des Arbeitskräftepotentials oder -angebots gemeinhin auch als eine Chance zur Verringerung der Erwerbslosigkeit betrachtet. Obwohl diese Sichtweise in der Tendenz zutreffend sein mag, hängt die zukünftige Entwicklung der Erwerbslosigkeit neben der Höhe des Arbeitsangebots noch von anderen Faktoren ab. Selbst in der einfachen Betrachtung des Arbeitsmarktes wird der Umfang der Erwerbslosigkeit eben nicht alleine durch das Angebot sondern auch durch die Entwicklung der Arbeitsnachfrage determiniert. Darüber hinaus ist Arbeitslosigkeit zumindest teilweise auch auf den qualifikatorischen und regionalen Mismatch zwischen Arbeitsangebot und -nachfrage zurückzuführen. Insofern sollte man sich davor hüten, aus dem demografischen Rückgang des Arbeitsangebots ohne eine eingehende-

\footnotetext{
${ }^{14}$ Als Datenbasis werden die durchschnittlichen Mikrozensus-Profile der Jahre 2005-2009 herangezogen und auf die VGR 2010 skaliert.
}

re Analyse gleichermaßen auf einen Rückgang der Erwerbslosigkeit und der Erwerbslosenquote zu schließen. Das vorliegende Szenario stellt daher lediglich eine Abschätzung des Arbeitszeiteffekts dar, den eine Halbierung der durchschnittlichen Erwerbslosenquote über alle Altersgruppen hinweg auf 3,5 Prozent im Jahr 2030 mit sich bringt. Um den Effekt isoliert betrachten zu können, verbleibt die Arbeitszeit je Erwerbstätigen und die Teilzeitarbeitsquote auf dem Ausgangsniveau des Jahres 2010.

\section{Arbeitszeitszenario 3: Erhöhung der Arbeitszeit Teilzeit- beschäftigter}

Da eine Steigerung der Arbeitszeit von den Beschäftigen selbst nur noch bei den teilzeitbeschäftigten Arbeitnehmern gewünscht ist (Wanger 2011, S. 6), wird im vorliegendem Szenario eine Erhöhung der Arbeitszeit für Teilzeitbeschäftigte um 50 Prozent bis 2030 modelliert. Die Teilzeitarbeitsquote sowie die Erwerbslosenquote bleiben auf dem Ausgangsniveau konstant.

\section{Arbeitszeitszenario 4: Senkung der Teilzeitbeschäftig- tenquote um ein Drittel}

Der Effekt, den eine Senkung der alters- und geschlechtsspezifischen Teilzeitquote um ein Drittel mit sich bringt, wird in Arbeitszeitszenario 4 analysiert. Die Umwandlung der Teilzeit- in Vollzeitjobs findet dabei wieder bis ins Jahr 2030 statt. Erwerbslosenquote und Arbeitszeit bleiben hingegen wieder konstant, um den isolierten Effekt betrachten zu können.

Arbeitszeitszenario 5: Halbierung der Erwerbslosenquote + Erhöhung der Arbeitszeit Teilzeitbeschäftigter Arbeitszeitszenario 5 stellt eine Kombination der Arbeitszeitszenarien 2 und 3 dar. Es findet somit eine Halbierung der Erwerbslosenquote bis 2030 statt, wobei im gleichen Zeitraum die Arbeitszeit der Teilzeitbeschäftigten um 50 Prozent ansteigt.

Arbeitszeitszenario 6: Halbierung der Erwerbslosenquote + Senkung der Teilzeitbeschäftigtenquote um ein Drittel

Arbeitszeitszenario 6 stellt eine Kombination der Arbeitszeitszenarien 2 und 4 dar. Es findet somit eine Halbierung der Erwerbslosenquote bis 2030 statt, wobei im gleichen Zeitraum ein Drittel der Teilzeit- in Vollzeitjobs umgewandelt wird.

Wie Tab. 5 zeigt, sinken die Arbeitsstunden je Erwerbstätigen pro Jahr unter den heutigen Gegebenheiten (Arbeitszeitszenario 1, Status Quo) bis ins Jahr 2060 hinein leicht ab. Dies ist auf die Altersverschiebung innerhalb der Erwerbsbevölkerung zurückzuführen, da ab einem Alter von 60 die Teilzeitquote für beide Geschlechter ansteigt und die 
Tab. 4 Übersicht der Arbeitszeitszenarien: Arbeitsvolumen pro Jahr

\begin{tabular}{|c|c|c|c|c|c|c|}
\hline & Arbeits & in Mio. & & & & \\
\hline & 2010 & 2020 & 2030 & 2040 & 2050 & 2060 \\
\hline Arbeitszeitszenario 1: Status Quo & 57087 & 55682 & 51049 & 47434 & 44605 & 41516 \\
\hline & $(0)$ & $(0)$ & $(0)$ & $(0)$ & $(0)$ & $(0)$ \\
\hline Arbeitszeitszenario 2: Halbierung ELQ & 57087 & 57100 & 53608 & 49827 & 46863 & 43606 \\
\hline & $(0)$ & (1418) & $(2560)$ & $(2393)$ & $(2258)$ & $(2090)$ \\
\hline Arbeitszeitszenario 3: & 57087 & 57637 & 54753 & 50866 & 47815 & 44520 \\
\hline Erhöhung Arbeitszeit Teilzeitbeschäftigter & $(0)$ & (1956) & $(3704)$ & $(3432)$ & $(3210)$ & (3004) \\
\hline Arbeitszeitszenario 4: & 57087 & 57306 & 54305 & 50400 & 47384 & 44135 \\
\hline Senkung Teilzeitbeschäftigtenquote um $1 / 3$ & $(0)$ & $(1625)$ & $(3256)$ & $(2965)$ & $(2779)$ & (2619) \\
\hline Arbeitszeitszenario 5: Szenario $2+3$ & 57087 & 59104 & 57492 & 53427 & 50231 & 46756 \\
\hline & $(0)$ & $(3422)$ & $(6444)$ & $(5992)$ & $(5626)$ & $(5240)$ \\
\hline Arbeitszeitszenario 6: Szenario $2+4$ & 57087 & 58764 & 57020 & 52936 & 49777 & 46350 \\
\hline & $(0)$ & $(3083)$ & $(5972)$ & $(5501)$ & $(5172)$ & $(4834)$ \\
\hline
\end{tabular}

Quelle: Eigene Berechnungen, Abweichungen vom Referenzszenario in Klammern

Tab. 5 Übersicht der Arbeitszeitszenarien: Arbeitszeit je Erwerbstätigen pro Jahr

\begin{tabular}{|c|c|c|c|c|c|c|}
\hline & \multicolumn{6}{|c|}{ Arbeitszeit je Erwerbstätigen in $h$} \\
\hline & 2010 & 2020 & 2030 & 2040 & 2050 & 2060 \\
\hline \multirow[t]{2}{*}{ Arbeitszeitszenario 1: Status Quo } & 1408 & 1395 & 1380 & 1383 & 1384 & 1382 \\
\hline & $(0)$ & $(0)$ & $(0)$ & $(0)$ & $(0)$ & $(0)$ \\
\hline \multirow[t]{2}{*}{ Arbeitszeitszenario 2: Halbierung ELQ } & 1408 & 1396 & 1380 & 1384 & 1384 & 1382 \\
\hline & $(0)$ & $(0)$ & $(0)$ & $(0)$ & $(0)$ & $(0)$ \\
\hline \multirow{2}{*}{$\begin{array}{l}\text { Arbeitszeitszenario 3: } \\
\text { Erhöhung Arbeitszeit Teilzeitbeschäftigter }\end{array}$} & 1408 & 1444 & 1480 & 1484 & 1483 & 1482 \\
\hline & $(0)$ & $(49)$ & $(100)$ & $(100)$ & $(100)$ & $(100)$ \\
\hline \multirow{2}{*}{$\begin{array}{l}\text { Arbeitszeitszenario } 4 \text { : } \\
\text { Senkung Teilzeitbeschäftigtenquote um } 1 / 3\end{array}$} & 1408 & 1436 & 1468 & 1470 & 1470 & 1469 \\
\hline & $(0)$ & $(41)$ & $(88)$ & $(86)$ & $(86)$ & $(87)$ \\
\hline \multirow[t]{2}{*}{ Arbeitszeitszenario 5: Szenario $2+3$} & 1408 & 1445 & 1480 & 1484 & 1484 & 1482 \\
\hline & $(0)$ & (49) & (100) & (100) & (100) & (100) \\
\hline \multirow[t]{2}{*}{ Arbeitszeitszenario 6: Szenario $2+4$} & 1408 & 1436 & 1468 & 1470 & 1470 & 1469 \\
\hline & $(0)$ & (41) & (88) & $(87)$ & (86) & (87) \\
\hline
\end{tabular}

Quelle: Eigene Berechnungen, Abweichungen vom Referenzszenario in Klammern

Zahl der in Teilzeit geleisteten Arbeitsstunden absinkt. Das Arbeitsvolumen der Erwerbstätigen pro Jahr verringert sich hierbei um ca. 30 Prozent auf 41,5 Millionen Stunden im Jahr 2060 (2010: 57 Millionen Stunden; vgl. Tab. 4). Insgesamt fällt der Rückgang des Arbeitsvolumens damit stärker aus als die Verringerung der Erwerbspersonenzahl. Eine Steigerung der Arbeitszeit von Teilzeitbeschäftigten um 50 Prozent (Arbeitszeitszenario 3) erhöht die Arbeitszeit je Erwerbstätigen bis 2030 um 5 Prozent (vgl. Tab. 5), und führt im Vergleich zum Status Quo zu einem Arbeitsvolumenzugewinn von ca. 3 Millionen Arbeitsstunden im Jahr 2060. Hingegen fällt der Arbeitsvolumeneffekt einer Halbierung der Erwerbslosenquote (Arbeitszeitszenario 2) im Mit- tel über die Jahre 2010 bis 2060 um 0,9 Millionen Arbeitsstunden kleiner aus. Der Arbeitsvolumeneffekt, der durch eine Umwandlung von einem Drittel der Teilzeit- in Vollzeitjobs ausgeht, liegt zwischen Arbeitszeitszenario 2 und 3. Hierbei sinkt das Arbeitsvolumen auf 44,1 Millionen Stunden im Jahr 2060 ab, wobei die Arbeitszeit je Erwerbstätigen gegenüber dem Jahr 2010 um ca. 4 Prozent ansteigt. Kombiniert man schließlich die Verringerung der Erwerbslosenquote (Arbeitszeitszenario 2) mit einer Erhöhung der Arbeitszeit (Arbeitszeitszenario 3) oder einer Senkung der Teilzeitquote (Arbeitszeitszenario 4), so steigt das Arbeitsvolumen im Vergleich zu den zugrunde liegenden Einzelszenarien für fast alle Jahre überproportional an. Dies ist letzt- 
Tab. 6 Bruttoinlandsprodukt je Einwohner bei konstanter Produktivität

\begin{tabular}{|c|c|c|c|c|c|c|}
\hline & \multicolumn{6}{|c|}{$\begin{array}{l}\text { BIP je Einwohner: } 2010=100 \text {; (jährliche Produktivitätswachstumsrate zur Stabilisierung des } \\
\text { BIP je Einwohner auf dem Niveau des Jahres } 2010 \text { in Prozent) }\end{array}$} \\
\hline & 2010 & 2020 & 2030 & 2040 & 2050 & 2060 \\
\hline \multirow[t]{2}{*}{ Arbeitszeitszenario 1: Status Quo } & 100.00 & 99.25 & 93.14 & 89.69 & 88.32 & 86.77 \\
\hline & $(0.00)$ & $(0.08)$ & $(0.36)$ & $(0.36)$ & $(0.31)$ & $(0.28)$ \\
\hline \multirow[t]{2}{*}{ Arbeitszeitszenario 2: Halbierung ELQ } & 100.00 & 101.78 & 97.81 & 94.22 & 92.79 & 91.14 \\
\hline & $(0.00)$ & $-(0.18)$ & $(0.11)$ & $(0.20)$ & $(0.19)$ & $(0.19)$ \\
\hline \multirow{2}{*}{$\begin{array}{l}\text { Arbeitszeitszenario 3: } \\
\text { Erhöhung Arbeitszeit Teilzeitbeschäftigter }\end{array}$} & 100.00 & 102.74 & 99.90 & 96.18 & 94.67 & 93.05 \\
\hline & $(0.00)$ & $-(0.27)$ & $(0.00)$ & $(0.13)$ & $(0.14)$ & $(0.14)$ \\
\hline \multirow{2}{*}{$\begin{array}{l}\text { Arbeitszeitszenario 4: } \\
\text { Senkung Teilzeitbeschäftigtenquote um } 1 / 3\end{array}$} & 100.00 & 102.15 & 99.08 & 95.30 & 93.82 & 92.25 \\
\hline & $(0.00)$ & $-(0.21)$ & $(0.05)$ & $(0.16)$ & $(0.16)$ & $(0.16)$ \\
\hline \multirow[t]{2}{*}{ Arbeitszeitszenario 5: Szenario $2+3$} & 100.00 & 105.35 & 104.90 & 101.02 & 99.46 & 97.72 \\
\hline & $(0.00)$ & $-(0.52)$ & $-(0.24)$ & $-(0.03)$ & $(0.01)$ & $(0.05)$ \\
\hline \multirow[t]{2}{*}{ Arbeitszeitszenario 6: Szenario $2+4$} & 100.00 & 104.75 & 104.04 & 100.10 & 98.56 & 96.88 \\
\hline & $(0.00)$ & $-(0.46)$ & $-(0.20)$ & $(0.00)$ & $(0.04)$ & $(0.06)$ \\
\hline
\end{tabular}

Quelle: Eigene Berechnungen

lich auf die zeitgleiche, schrittweise Einführung einer höheren Erwerbstätigenzahl in Kombination mit einer höheren Arbeitszeit je Erwerbstätigen zurück zu führen.

Die dargestellten Arbeitsvolumenberechnungen lassen sich unter der Annahme einer konstanten Arbeitsproduktivität je Erwerbstätigenstunde in die reale Bruttoinlandsproduktentwicklung je Einwohner überführen. Die erste Zeile des jeweiligen Arbeitszeitszenarios in Tab. 6 zeigt dabei, dass die Arbeitszeitszenarien 3 und 4 eine annähernde Stabilisierung des Bruttoinlandsprodukt je Einwohner bis zum Jahr 2030 ermöglichen, wohingegen in den Kombinationsszenarien 5 und 6 eine Zunahme des Bruttoinlandsprodukts je Einwohner sogar bei Vernachlässigung zukünftiger Produktivitätszuwächse möglich erscheint. Hingegen fällt das Bruttoinlandsprodukt je Einwohner im Jahr 2030 auf 93,1 Prozent des Ausgangsniveaus des Jahres $2010 \mathrm{ab}$, sollte über die extensive Arbeitsangebotserhöhung - die durch das Basisszenario der Erwerbspersonenprojektion modelliert wird - hinaus keine Anpassung des intensiven Arbeitsangebots oder der Erwerbslosenquote erfolgen (Arbeitszeitszenario 1). Dies würde letztlich auch bedeuten, dass 0,36 Prozentpunkte des jährlichen Produktivitätswachstums im Zeitraum der Jahre 2010 bis 2030 allein zur Stabilisierung des Bruttoinlandsprodukt je Einwohner benötigt würden. Dies entspricht in etwa einem Drittel der jährlichen Zuwachsrate der totalen Faktorproduktivität, die im Durchschnitt der Jahre 1992 bis 2010 bei ca. einem Prozent lag (Sachverständigenrat zur Begutachtung der gesamtwirtschaftlichen Entwicklung 2011, S. 136). Wie vorliegende Modellrechnungen jedoch auch zeigen, wird mit jeder weiteren Erhöhung des Arbeitsangebots, das zur Wohlstandssicherung benötigte Produktivitätswachstum gedrückt, so dass beispielsweise alleine eine Halbierung der Erwerbslosenquote bis ins Jahr 2030 die benötigte jährliche Produktivitätswachstumsrate auf 0,11 Prozent senkt. Im Zeitraum nach 2030 wird hingegen das abnehmende Erwerbspersonenpotential zunehmend deutlich, so dass lediglich in den Arbeitszeitszenarien 5 und 6 eine annähernde Stabilisierung des Bruttoinlandsprodukt je Einwohner bis ins Jahr 2060 gelingt. Allerdings muss im Hinblick auf die Entwicklung nach 2030 berücksichtigt werden, dass im vorliegenden Beitrag keine über das Jahr 2030 hinausreichenden Maßnahmen berücksichtigt wurden.

\section{Fazit}

Die Ergebnisse der Erwerbspersonenprojektion des vorliegenden Beitrags reihen sich in den bisherigen Forschungsstand ein. Sie liegen dabei am oberen Rand, was zum einen auf die relativ optimistische Annahme bezüglich der zukünftigen Nettozuwanderung, ${ }^{15}$ und zum anderen auf die günstige Ausgangslage bezüglich der Erwerbsbeteiligung im Basisjahr zurückzuführen sein dürfte. Darüber hinaus könnte sich insbesondere die Fortschreibung mit den über die Zeit konstanten Entry- und Exit-Rates als zu statisch erweisen.

\footnotetext{
${ }^{15}$ Den jeweiligen Primär- bzw. Basisvarianten der Berechnungen des Statistischen Bundesamtes (2009b), jenen von Bonin et al. (2011) sowie jenen von Werding (2011) liegt die Untergrenze der mittleren Bevölkerung zu Grunde (Außenwanderungssaldo: +100.000). Börsch-Supan und Wilke (2009, S. 36) gehen in ihrem Basisszenario der Bevölkerungsprojektion von einem Außenwanderungssaldo von +150.000 aus. Dies trifft auch auf die Erwerbspersonenprojektionen von Bomsdorf (2008, S. 142) zu.
} 
Dies zeigt sich beispielsweise bei den weiblichen Erwerbsquoten, die bis ins Jahr 2009 hinein von der 1992 angestoßenen Rentenreform beeinflusst sind und deren Fortschreibung daher leicht verzerrt sein dürfte. Letztlich wohnt dieses Problem aber auch den Berechnungen von (Werding 2011) inne, so dass sich die durchschnittliche Abweichung der hier vorgestellten Berechnungen im Vergleich zu (Werding 2011) im Wesentlichen aus den unterschiedlichen Annahmen zur Höhe des Wanderungssaldos und der günstigeren Ausgangslage bezüglich der Erwerbsbeteiligung im Basisjahr ergeben. ${ }^{16}$

Trotz der vergleichsweise optimistischen Annahmen ergibt sich im Basisszenario ein Rückgang der Erwerbsbevölkerung um 9 Prozent bis 2030 und um 26 Prozent bis 2060. Gleichzeitig haben die verschiedenen Modellrechnungen gezeigt, dass noch weitere, bisher unausgeschöpfte Potentiale bestehen, um diese Lücke zu schließen. Die größten Effekte sind dabei bei der Verlängerung der Lebensarbeitszeit und einer generell höheren Erwerbsbeteiligung von Älteren zu erzielen. Der Effekt eines früheren Erwerbseintritts ist im Vergleich hierzu zwar eher gering, kann jedoch aufgrund der bereits bestehenden Gesetzeslage als gegeben angesehen werden. Hingegen birgt gerade die Förderung von Frauen und hier insbesondere von Frauen mit Migrationshintergrund noch ausschöpfbare Möglichkeiten, die in $\mathrm{Zu}-$ kunft weiter gefördert werden sollten.

Gleichzeitig haben die Ergebnisse des vorliegenden Beitrags gezeigt, dass eine Stabilisierung des absoluten Arbeitsvolumens aus dem Jahr 2010 bis ins Jahr 2030 hinein möglich ist, wenn es flankierend zur Förderung der Erwerbsbeteiligung gelingt, die Erwerbslosenquote zu reduzieren und die geleisteten Arbeitsstunden je Erwerbstätigen zu erhöhen. Dies könnte beispielsweise durch eine Verringerung der Teilzeitquote oder aber eine Erhöhung der Arbeitszeit der Teilzeitbeschäftigten geschehen. Die Implikationen, die diese nominelle Stabilisierung des Arbeitsvolumens auf das Bruttoinlandsprodukt je Einwohner hat, sind aufgrund der fallenden Einwohnerzahl positiv und erlauben selbst bei Vernachlässigung zukünftiger Produktivitätszuwächse eine Steigerung des Bruttoinlandsprodukts je Einwohner. Sollte es hingegen nicht gelingen, die Erwerbslosenquote zu senken oder die geleisteten Arbeitsstunden je Erwerbstätigen zu erhöhen, so ist damit zu rechnen, dass ein Drittel des zukünftigen Produktivitätswachstums zur Aufrechterhaltung des heutigen Wohlstandniveaus nötig ist.

Zusammenfassend lässt sich festhalten, dass die Herausforderungen des demografischen Wandels bezüglich der Erwerbspersonenzahl und des Arbeitsvolumens bis ins Jahr

\footnotetext{
${ }^{16}$ Die in vorliegender Arbeit berechnete Zahl der Erwerbspersonen liegt im Durchschnitt für die Jahre 2010 bis 2060 um 530.000 Personen höher, als jene von Werding (2011). Vgl. hierzu auch Sachverständigenrat zur Begutachtung der gesamtwirtschaftlichen Entwicklung (2011, S. 94).
}

2030 hinein zu meistern sind. Gleichzeitig legen die Ergebnisse nahe, dass die Auswirkungen des demografischen Wandels im Zeitraum nach 2030 zu einer deutlichen Verringerung der Erwerbspersonenzahl und des Arbeitsvolumens führen werden. Allerdings ist bei der Interpretation der Ergebnisse für die lange Frist zu berücksichtigen, dass selbst bei Umsetzung der im vorliegenden Beitrag betrachteten Szenarien noch weitere Potentiale zur Erhöhung der Erwerbsbeteiligung bestehen und auch die Möglichkeiten der Zuwanderung zur Sicherung des Arbeitskräftepotentials nicht betrachtet wurden.

\section{Kurzfassung}

Der vorliegende Beitrag zeigt anhand verschiedener Simulationsrechnungen, welche Handlungsfelder grundsätzlich geeignet erscheinen, dem demografisch bedingten Arbeitskräfterückgang zu begegnen. Aufbauend auf einer Bevölkerungsvorausberechnung, die sich an den Annahmen der 12. koordinierten Bevölkerungsvorausberechnung des Statistischen Bundesamtes orientiert, werden in einem ersten Schritt verschiedene Erwerbspersonenprojektion vorgenommen: Dabei werden neben einer kohortenspezifischen Fortschreibung der Erwerbsbeteiligung gemäß der im Mikrozensus beobachteten Trends die Konsequenzen einer Erhöhung des effektiven Renteneintrittsalters, eines früheren Erwerbseintritts, einer steigenden Erwerbsbeteiligung der über 60-Jährigen, einer verstärkten Erwerbsbeteiligung von Menschen mit Migrationshintergrund sowie einer erhöhten Erwerbsbeteiligung der Frauen untersucht. In einem zweiten Schritt werden die Auswirkungen einer steigenden Jahresarbeitszeit sowie einer sinkenden Erwerbslosenquote auf das jährlich geleistete Arbeitsvolumen betrachtet. Bei unterstellter Konstanz der Arbeitsproduktivität lassen die Arbeitsvolumenprojektionen hierbei auch Rückschlüsse über das zukünftige Niveau des Bruttoinlandsprodukts je Einwohner zu.

Gemäß dem Basisszenario der Erwerbspersonenprojektion, welches neben den kohortenspezifischen Trends zusätzlich auch die Erhöhung der Regelaltersgrenze auf 67 Jahre berücksichtigt, bleibt festzuhalten, dass der Anteil der Erwerbspersonen an der Gesamtbevölkerung bis zum Jahr 2020 in etwa auf dem heutigen Niveau aufrecht erhalten werden kann, bis zum Jahr 2030 jedoch um etwa 3 Prozentpunkte abfällt. Um den demografisch bedingten Rückgang des Arbeitskräfteangebots zu kompensieren, werden daher noch weitere Maßnahmen notwendig sein. Ein großes Potential bieten hierbei insbesondere Maßnahmen, die auf eine Erhöhung der Erwerbsbeteiligung Älterer abzielen. So würde die Erhöhung des effektiven Renteneintrittsalters um ein weiteres Jahr die Lücke im Jahr 2030 auf 1,6 Prozentpunkte verkleinern und die Erhöhung der Erwerbsquote der 60 bis 65-Jährigen auf das Niveau der 55 bis 60-Jährigen die Lücke 
annähernd schließen. Hingegen ergibt sich durch die Absenkung des Erwerbseintrittsalters um ein Jahr nur ein vergleichsweise geringer Effekt. Der Effekt einer Angleichung der Erwerbsbeteiligung von Menschen mit Migrationshintergrund an diejenige von Menschen ohne Migrationshintergrund könnte die Lücke im Jahr 2030 um etwa einen Prozentpunkt schließen. Dies entspricht in etwa dem Effekt, welcher sich aus einer Halbierung des Abstands zwischen der Erwerbsbeteiligung von Frauen und Männern erzielen lässt. Obwohl das Potential eines einzelnen Handlungsfeldes für sich genommen von Interesse ist, wird eine effektive Strategie zur Sicherung des Arbeitskräftepotentials an mehreren der bereits angesprochenen Handlungsfelder ansetzen müssen. Der vorliegende Beitrag betrachtet daher abschlieBend die Auswirkungen eines Kombinationsszenarios. Im Vergleich zu den Einzelszenarien kann die Lücke bis zum Jahr 2030 hierbei nicht nur geschlossen sondern der Anteil der Erwerbspersonen an der Gesamtbevölkerung gegenüber dem Jahr 2010 um 1,5 Prozentpunkte erhöht werden. Nach dem Jahr 2030 sinkt dieser Anteil jedoch in allen Szenarien deutlich ab. Dies ist zum einen auf das sinkende Erwerbspersonenpotential und zum anderen darauf zurückzuführen, dass die betrachteten Szenarien nach 2030 keine weitere Anpassung der Erwerbsbeteiligung vorsehen.

Da sich die Betrachtung der Erwerbspersonen alleine auf die Anzahl der am Arbeitsmarkt aktiven Personen beschränkt, wird für das Basisszenario der Erwerbspersonenprojektion in einem zweiten Schritt die Entwicklung der geleisteten Arbeitsstunden bzw. des Arbeitsvolumens untersucht. Als mögliche Szenarien werden hierbei eine Halbierung der Erwerbslosenquote sowie eine Anhebung der Arbeitszeit je Erwerbstätigen - mittels einer Erhöhung der Arbeitszeit Teilzeitbeschäftigter um 50 Prozent oder einer Absenkung der Teilzeitbeschäftigtenquote um ein Drittel betrachtet. Die Ergebnisse zeigen zunächst, dass das Arbeitsvolumen unter den heutigen Gegebenheiten bis zum Jahr 2030 stärker zurückgehen wird, als die Zahl der Erwerbspersonen. Dies ist im Wesentlichen auf die höhere Teilzeitquote von Frauen und Älteren zurückzuführen. Hingegen lässt sich durch die Kombination einer sinkenden Erwerbslosenquote mit einer Erhöhung der Arbeitszeit je Erwerbstätigen eine Stabilisierung des Arbeitsvolumens bis zum Jahr 2030 auf dem Niveau des Jahres 2010 erreichen.

In einem dritten Schritt untersucht der vorliegende Beitrag schließlich die Implikationen für die Entwicklung des Bruttoinlandsprodukts je Einwohner. Unter der Annahme einer konstanten Arbeitsproduktivität zeigen die Ergebnisse für das Basisszenario der Erwerbspersonenprojektion, dass eine Erhöhung der Arbeitszeit Teilzeitbeschäftigter um 50 Prozent zur Stabilisierung des Bruttoinlandsprodukts je Einwohner ausreichen würde. Eine Halbierung der Erwerbslosenquote oder die Absenkung der Teilzeitbeschäftigtenquote um ein Drittel bis zum Jahr 2030 sind hingegen nicht ausreichend, um dieses Ziel zu erreichen. Schließlich könnte durch eine Kombination aus einer Erhöhung der Arbeitszeit je Erwerbstätigen mit einer Halbierung der Erwerbslosenquote - selbst bei Vernachlässigung zukünftiger Produktivitätssteigerungen - sogar eine Erhöhung des Bruttoinlandsprodukts je Einwohner bis zum Jahr 2030 erreicht werden.

Zusammenfassend lässt sich festhalten, dass bereits die heute beobachtbaren Veränderungen im Erwerbsverhalten der Frauen und der Älteren sowie die politische Weichenstellung durch die Rente mit 67 ausreichend sein dürften, um das Arbeitsangebot bis zum Jahr 2020 auf dem heutigen Niveau zu stabilisieren. Hingegen wird die Sicherung des Fachkräftebedarfs der Wirtschaft bis zum Jahr 2030 weitergehende Anstrengungen erfordern. Insgesamt sind die Herausforderungen des demografischen Wandels für den Arbeitsmarkt zumindest bis zum Jahr 2030 jedoch zu bewältigen.

\section{Executive summary}

Based on a scenario approach this paper analyzes various policy options to counteract the demographic decline in labor supply. The starting point of our analysis is a population forecast which follows the assumptions of the 12th official population projection of the Federal Statistical Office in Germany. Based on our population forecast we conduct several labor force forecasts: First, to account for observable trends in labor force participation our trend scenario applies a cohort-component model to derive future participation rates by age and sex. Augmenting the trend scenario to account for the impact of the legislated rise in the legal retirement age on the participation rates of the elderly gives our baseline scenario. Second, based on the baseline scenario we assess the impact of various policy options to counteract the demographic decline in the labor force. Our menu of policy experiments includes an increase in the effective retirement age, a decrease in the age of labor market entry, an increase in the labor force participation of the elderly, an increase in the labor force participation of immigrants as well as an increase in female participation rates. Third, we analyze the impact of demographic change on the annual volume of work and GDP (per capita).

Despite a rise in the labor force participation of females and elderly the results of our baseline scenario suggest that overall labor force participation will decline by 3 percentage points until 2030 due to population ageing. Therefore further measures are necessary to compensate for the demographic decline in labor supply. Obviously all measures that aim at increasing the participation rates of the elderly offer a huge potential. An increase in the effective retirement age by one more year will close the gap in 2030 by 1.4 percentage points, while an increase in the labor force participation of people aged 60 to 65 to the level of people aged 55 to 60 will 
almost close the gap in 2030. This contrasts sharply with the very small effect of a decline in the labor market entry age by one year. The impact of raising the labor force participation of people with a migration background is of similar magnitude as the effect of a decline in the labor force participation gap between men and women by 50 percent and amounts to 1 percentage point. Even though the potential of a single policy option is of interest, a holistic approach will be needed in the future. Therefore a combination scenario is considered taking all mentioned policy options into account. Comparing the outcome of the combination scenario with all other scenarios yields an interesting result: This scenario is not only able to close the gap in 2030 but shows an increase in overall labor force participation by 1.5 percentage points compared to 2010 . However this share decreases in all scenarios after 2030. The reasons are obvious: On the one hand the labor force potential is declining, while on the other hand no further adjustments in the labor force participation rate are considered.

As the examination of the labor force does only account for the number of people participating in the labor market, we focus on the hours worked by those people and the annual volume of work in the second part of our paper. The labor force projection used in this part is hence based on the baseline scenario. As possible settings we consider a 50 percent decline in the unemployment rate as well as an increase in the hours worked by each employee. The increase in the hours worked by each employee is implemented in two ways: First by a 50 percent increase for people who are part time employed, second by a one third decrease in the part time employee rate. Our results show that the annual volume of work is going to decline faster than the labor force until 2030 if nothing is changed. This is mainly because women and elderly work more part time than the remaining population. If it is however possible to combine a declining unemployment rate with an increase in the hours worked a stabilization of the 2010 annual volume of work is within reach.

The third part of our paper is concerned with the implications our forecasts of the volume of work have for GDP per capita. Taking labor productivity as given, our results suggest that a stabilization of the GDP per capita is possible by increasing the hours worked by part time employees by 50 percent. However a decrease in the unemployment rate by 50 percent or a decrease in the part time employee rate by one third is not sufficient. In contrast, a combination of a decreasing unemployment rate and an increase in the hours worked leads to an increase in GDP per capita until 2030 even without any changes in productivity.

Danksagung Ein besonderer Dank geht an die Mitglieder der Arbeitsgruppe „Arbeitsmarkt“, die sich im Rahmen des Projekts ,Zukunft der Arbeitswelt" der Robert-Bosch-Stiftung zusammen fand. Namentlich sind hierbei Lutz Bellmann, Eckart Bomsdorf, Werner Eichhorst,
Silke Eilers, Hans-Peter Klös sowie Matthias Schuster zu nennen. Für weitere Hinweise danken wir zwei anonymen Gutachtern sowie Johannes Vatter. Alle verbleibenden Fehler sind die der Autoren.

\section{Literatur}

Antuofermo, M., Meglio, E.: $23 \%$ of EU citizens were at risk of poverty or social exclusion in 2010. Statistics in Focus 9/2012, Eurostat (2012)

Bomsdorf, E.: Arbeitskräftepotential und demografischer Wandel. Modellrechnungen für die Bundesrepublik Deutschland bis 2050. Jahrb. Wirtsch.gesch. 49(1), 123-146 (2008)

Bonin, H.: Generational Accounting: Theory and Application. Springer, Berlin (2001)

Bonin, H., Kappler, S., Sachs, A.: Wertschöpfungseffekte der Fachkräftesicherung. ZEW Forschungsbericht, Mannheim (2011)

Börsch-Supan, A.: Labor market effects of population aging. Labour 17, 5-44 (2003)

Börsch-Supan, A., Wilke, C.: Zur mittel-und langfristigen Entwicklung der Erwerbstätigkeit in Deutschland. Z. Arb.markt Forsch. 42(1), 29-48 (2009)

Bundesregierung: Nationaler Aktionsplan Integration: Zusammenhalt stärken - Teilhabe verwirklichen. Presse- und Informationsamt der Bundesregierung, Berlin (2011)

Burniaux, J., Duval, R., Jaumotte, F.: Coping with ageing: a dynamic approach to quantify the impact of alternative policy options on future labour supply in OECD countries. OECD Economics Department working papers 371, OECD Publishing (2004)

Deutsche Rentenversicherung: Rentenversicherung in Zeitreihen. DRV-Schriften 22, DRB, Berlin (2011)

Europäische Kommission: The 2005 projections of age-related expenditure (2004-50) for the EU-25 member states: underlying assumptions and projection methodologies. Special report 4/2005, European Communities, Brussels (2006)

Fuchs, J., Söhnlein, D.: Lassen sich die Erwerbsquoten des Mikrozensus korrigieren. IAB Werkstattbericht 12/2003, Institut für Arbeitsmarkt- und Berufsforschung, Nürnberg (2003)

Keese, M.: A method for calculating the average effective age of retirement. OECD (2007). http://www.oecd.org/dataoecd/3/0/ 39371923.pdf. Stand: 09.10.2012

Rengers, M.: Das international vereinbarte Labour-Force-Konzept. Wirtsch. Stat. 12(2004), 1369-1383 (2004)

Sachverständigenrat zur Begutachtung der gesamtwirtschaftlichen Entwicklung: Herausforderungen des demografischen Wandels. Statistisches Bundesamt, Wiesbaden (2011)

Scherer, P.: Age of withdrawal from the labour force in OECD countries. OECD Labour Market and Social Policy Occasional papers 49, OECD Publishing (2002)

Statistisches Bundesamt: Bevölkerung Deutschlands Bis 2060 12. Koordinierte Bevölkerungsvorausberechnung. Statistisches Bundesamt, Wiesbaden (2009a)

Statistisches Bundesamt: Demografischer Wandel in Deutschland Auswirkungen Auf die Zahl der Erwerbspersonen, Heft 4. Statistisches Bundesamt, Wiesbaden (2009b)

Statistisches Bundesamt: Bevölkerung und Erwerbstätigkeit Bevölkerung Mit Migrationshintergrund - Ergebnisse des Mikrozensus 2010-. Fachserie 1, Reihe 2.2. Statistisches Bundesamt, Wiesbaden (2011a)

Statistisches Bundesamt: Volkswirtschaftliche Gesamtrechnung. Statistisches Bundesamt, Wiesbaden (2011b)

Wanger, S.: Ungenutzte Potentiale in der Teilzeit - Viele Frauen würden gerne länger arbeiten. IAB-Kurzbericht 9/2011, Institut für Arbeitsmarkt- und Berufsforschung, Nürnberg (2011) 
Werding, M.: Demographie und öffentliche Haushalte - Simulationen zur langfristigen Tragfähigkeit der gesamtstaatlichen Finanzpolitik in Deutschland. Arbeitspapier 3/2011, Sachverständigenrat zur Begutachtung der gesamtwirtschaftlichen Entwicklung, Wiesbaden (2011)

Werding, M., Hofmann, H.: Projektionen zur Langfristigen Tragfähigkeit der Öffentlichen Finanzen. Ifo Beiträge zur Wirtschaftsforschung, Bd. 30. ifo Institut, München (2008)

Daniel Ehing Dipl.-Vw., Studium der Volkswirtschaftslehre an der Albert-Ludwigs-Universität Freiburg i.Br. und der University of British Columbia (Vancouver). Seit 2011 wissenschaftlicher Mitarbeiter am Institut für Volkswirtschaftslehre und Finanzwissenschaft I und am Forschungszentrum Generationenverträge an der Albert-LudwigsUniversität Freiburg i.Br.

Stefan Moog Dipl.-Vw., Studium der Volkswirtschaftslehre an der Albert-Ludwigs-Universität Freiburg i.Br. und dem Studienzentrum Gerzensee (Schweiz). Seit 2001 wissenschaftlicher Mitarbeiter am Institut für Volkswirtschaftslehre und Finanzwissenschaft I und am Forschungszentrum Generationenverträge an der Albert-LudwigsUniversität Freiburg i.Br. Seine Forschungsschwerpunkte liegen in den Bereichen der dynamischen Fiskalpolitik, der Verteilungstheorie und der fiskalischen Nachhaltigkeitsanalyse. 